\title{
3. What Does Neuroethics Have to Say about the Problem of Dual Use?
}

\author{
Valentina Bartolucci and Malcolm Dando
}

\section{Introduction}

It is clear that in the past advances in neuroscience were used for hostile as well as peaceful purposes. Lethal chemical nerve agents, after all, interfered with the acetylcholine neurotransmitter system ${ }^{1}$ and, during the twentieth century's East-West Cold War, both sides clearly also made efforts to develop 'nonlethal' chemical agents for various purposes. ${ }^{2}$ The use of some form of fentanyl derivative(s) by Russian security forces to break the 2002 Moscow theatre siege shows that today at least one major state has deployed such a weapons system. Many commentators fear that Russia would be far from alone in having an interest in developing novel incapacitating capabilities if the advances in neuroscience provide suitable opportunities. ${ }^{3}$

This chapter sketches the areas of interest and methods used by neuroethicists to ask what they have had to say about the problem of dual use: the fact that advances in benignly intended civil neuroscience could produce materials, knowledge and technologies that might then be used for hostile purposes by others. Of course, it should be understood from the start that this is no small problem, as has been made abundantly clear, for example, in the Lemon-Relman report of the US National Academies in 2006, which, in its second recommendation, stated that it was necessary to "[a]dopt a broadened awareness of threats beyond the classical "select agents" and other pathogenic organisms and toxins, so as to include, for example, approaches for disrupting host homeostatic and defence systems and for creating synthetic organisms'. ${ }^{4}$

1 Dando, M. R. 1996, A New Form of Warfare: The Rise of Non-Lethal Weapons, Brassey's, London.

2 Dando, M. R. and Furmanski, M. 2006, 'Midspectrum incapacitant programs', in M. L. Wheelis, L. Rózsa and M. R. Dando (eds), Deadly Cultures: Biological Weapons Since 1945, Harvard University Press, Cambridge, Mass., pp. 236-51.

3 Pearson, A. M., Chevrier, M. and Wheelis, M. L. 2007, Incapacitating Biochemical Weapons: Promise or Peril? Lexington Books, Lanham, Md.

4 Committee on Advances in Technology and the Prevention of their Application to Next Generation Biowarfare Threats 2006, Globalization, Biosecurity and the Future of the Life Sciences, The National Academies Press, Washington, DC. 
Host homeostatic and defence systems obviously would include hormones of the endocrine system, neurotransmitters of the nervous system and cytokines of the immune system - understanding all of which is critical to our continuing advances in neuroscience.

\section{The rise of neuroethics}

As Robert Blank argued before the turn of the century, whilst there may be some issues unique to the societal impacts of neuroscience, the issues raised by advances in this area are basically similar to those raised in other areas of medical advances. ${ }^{5}$ He suggested that there are, in fact, three clear levels of policy dimensions in regard to all of these areas of technology:

1.... [D]ecisions must be made concerning the research and development of the technologies ...

2. The second policy dimension relates to the individual use of technologies once they are available ...

3. The third dimension of biomedical policy centers on the aggregate societal consequences of widespread application of a technology. ${ }^{6}$

These roots within the general growth of bioethical concerns are generally acknowledged by neuroethicists. As Raymond de Vries noted in the 2007 special issue of EMBO Reports on 'The Biology of Behaviour: Scientific and Ethical Implications':7 'Most histories of neuroethics are varieties of the technology story. Illes and Bird (2006) place the history of neuroethics squarely in the standard account of bioethics that runs from the Nuremberg Code in 1947, to the 1964 Declaration of Helsinki ... to the Belmont Report in 1979.'

The technology story, de Vries explains, is one in which new technologies bring ethical questions that are too difficult for ordinary practitioners to answer and they therefore need expert guidance from ethicists. Being a sociologist, de Vries does not think this is the only possible history, but it is one that will be here accepted for the moment.

Despite the acknowledgment of these long roots within bioethics, it appears to be widely accepted that, as a particular field of study, 'neuroethics' originated

\footnotetext{
5 Blank, R. H. 1999, Brain Policy: How the New Neuroscience Will Change Our Lives and Politics, Georgetown University Press, Washington, DC.

6 Ibid., pp. 11 and 12.

7 de Vries, R. 2007, 'Who will guard the guardians of neuroscience? Firing the neuroethical imagination', EMBO Reports, vol. 8 (S1), pp. S65-9.
} 
after the turn of the century. Martha Farah, ${ }^{8}$ in a thoughtful attempt to delineate the field, argued, for example, that '[b]eginning in 2002, neuroscientists began to address these issues in the scientific literature ... and the field gained a name "neuroethics". At the same time, key meetings brought together large numbers of experts and specific neuroethics membership organisations began to be founded. ${ }^{\prime 9}$

The comparatively recent delineation of neuroethics as a special field it is not too surprising; however, given its acknowledged deep roots within bioethics, as Parens and Johnston carefully pointed out, it is important to understand that bioethicists have made major errors in the past. If neuroethicists forget past mistakes they could end up doing the same in the near future. ${ }^{10}$ Parens and Johnston point to three particular problems that could easily arise in the new field of neuroethics:

1.... [T] he problem of reinventing the bioethical wheel ...

2.... [T] problem of exaggerating what the science can teach us about who we are...

3. ... [T] he problem of exaggerating what bioethics research can deliver. ${ }^{11}$

Furthermore, given the recent delineation of the specific field of neuroethics, we should not be surprised to find that there is as yet no comprehensive, widely accepted view of the scope of the field. For example, one might argue that finding out what happens in the brain when ethical decisions are being made should be central to any conception of neuroethics, but that does not appear to be what practitioners have decided to do. Rather, the field seems to deal more with what Farah called 'the practical and the philosophical': the implications of advances in neuroscience for practical social issues and the implications of advances in neuroscience for our understanding of ourselves (which, of course, overlaps to some extent with the question of how we make ethical decisions). The sections of Farah's paper provide illustrations of these different aspects (Table 3.1).

\footnotetext{
8 Farah, M. J. 2005, 'Neuroethics: the practical and the philosophical', Trends in Cognitive Sciences, vol. 9, no. 1, pp. 34-40, see Figure 3.1: 'Milestones in the history of ethics in neuroscience'.

9 See, for example, 'The History of Neuroethics' section of the entry on 'Neuroethics' in Wikipedia.

10 Parens, E. and Johnston, J. 2007, 'Does it make sense to speak of neuroethics?' EMBO Reports, vol. 8

(S1), pp. S61-4.

11 From Wikipedia, op. cit., p. 64.
} 
On the Dual Uses of Science and Ethics

\section{Table 3.1 Neuroethics: Practical and Philosophical}

Practical: Brain imagining and brain privacy

- Among the neuroscience technologies that present new ethical challenges of a practical nature is functional brain imaging.'

- 'For example, in "neuromarketing" brain imaging is used to measure limbic system response to a product that may indicate consumer's desire for it.'

Philosophical: Science and the soul

- 'Recent neuroimaging research has shown a characteristic pattern of brain activation associated with states of religious transcendence, which is common to Buddhist meditation and Christian prayer.'

- 'The idea that there is somehow more to a person than their physical instantiation runs deep in the human psyche and is a central element in virtually all the world's religions.'

- 'Neuroscience has begun to challenge this view, by showing that not only perception and motor control, but also character, consciousness and sense of spirituality may all be features of the machine.'

Source: de Vries, R. 2007, 'Who will guard the guardians of neuroscience? Firing the neuroethical imagination', EMBO Reports, vol. 8 (S1), pp. S65-9.

Neil Levy, in his introduction to the new journal Neuroethics, accepted this twofold division of the subject but stressed that, to the extent that neuroscience shows that we are less than rational and autonomous in our decision-making, that must impact on our understanding of the practical impact of the advances in neuroscience. ${ }^{12}$

In an attempt to advance the field of neuroethics, Georg Northoff argued that both of Farah's two subdivisions of neuroethics (which might be simply characterised as what we can do and what we know) should be termed 'empirical neuroethics', and we should accept that there is no sharp distinction between them (Table 3.2). So, in his view: 'Empirical neuroethics deals with the empirical and practical aspects of the linkage between neuroscientific and ethical concepts.' ${ }^{13}$

12 Levy, N. 2008, 'Introducing neuroethics', Neuroethics, vol. 1, pp. 1-8.

13 Northoff, G. 2009, 'What is neuroethics: empirical and theoretical neuroethics', Current Opinion in Psychiatry, vol. 22, pp. 565-9. 
Table 3.2 Aspects of Empirical Neuroethics

\begin{tabular}{|lll|}
\hline Technology & $\begin{array}{l}\text { Questions } \\
\text { Old technology }\end{array}$ & New questions \\
\cline { 2 - 3 } & Safety & Intentional deception \\
& $\begin{array}{l}\text { Researchers' } \\
\text { obligations }\end{array}$ & Neuromarketing \\
& Validity & $\begin{array}{l}\text { Personal } \\
\text { characteristics }\end{array}$ \\
Pharmacological & Safety & Attention \\
enhancement & Validity & Memory \\
& & Mood \\
& & Equity \\
BMI and N-P & & Military research on \\
enhancement & & cyborgs \\
\hline
\end{tabular}

Source: Northoff, G. 2009, 'What is neuroethics: empirical and theoretical neuroethics', Current Opinion in Psychiatry, vol. 22, pp. 565-9.

He writes, however, that '[a]lthough there has been much discussion of various issues in empirical neuroethics, the discussion of methodological and conceptual issues and thus theoretical neuroethics has remained rather sparse so far'.

Thus, Northoff argues for the need for theoretical neuroethics to focus 'on the methodological and conceptual aspects' of the linkage between neuroscientific facts and ethical concepts and for a theoretical neuroscience that is able to give proper weight to both norms and facts in a 'norm-fact circularity'.

Towards the end of his paper, Northoff notes that his ideas may strike some as a mere playground for theoreticians - a criticism that might be particularly applied to stressing theoretical neuroscience in this paper on the severely practical issue of dual use. We think, however, he has a general point to make on neuroethics methodology that is significant: 'If neuroethics wants to establish itself as a separate discipline that is different from its neighboring disciplines like philosophy, ethics and neuroscience, it must develop a special methodology.'

In this view, a discipline or field of study cannot claim to be distinct just because it studies certain things; it must have developed its own distinctive methodology as well. It would certainly be wrong to consider that this will be a simple task given that there are still great philosophical differences about how we should go about understanding our brains and behaviour. ${ }^{14}$

14 Evers, K. 2007, 'Towards a philosophy for neuroethics', EMBO Reports, vol. 8, pp. 848-51. 
Although the recent conceptualisation of neuroethics originated in the United States and Europe, research and publications on neuroethics are increasingly international. Lombera and Illes suggested that despite the broad scope of the field, "neuroethics has attempted to frame its efforts in terms of four "pillars": brain science and the self, brain science and social policy, ethics and the practice of brain science, and brain science and public discourse' ${ }^{15}$

On this basis, they carried out a wide-ranging literature survey and concluded that their results demonstrated 'a steady increase in global participation in neuroethics from 1989 to 2005, characterized by an increase in numbers of articles published specifically on neuroethics, journals publishing these articles, and countries contributing to the literature' - and they clearly expect this trend to continue as neuroscience advances and the associated technologies spread around the world.

Neuroscientists and neuroethicists have been commendably interested in the problem of communication with the public about their work ${ }^{16}$ and about how neuroscientists might be better enabled to carry out such tasks. Yet there are also reasons to believe that neuroscientists do not readily take to ethical analyses and the communication of their ethical positions, ${ }^{17}$ and that they are often not trained to do so. ${ }^{18}$ This, of course, is cause for considerable concern because, as the sociologist Raymond de Vries pointed out, there are other possible accounts of the rise of bioethics besides the technology story. For example, de Vries cites one account in which '[u]nlike the conventional technology story, in which bioethicists are cast as the guardians who oversee and regulate doctors and scientists ... bioethicists are less-than-critical allies of medicine and medical science'. ${ }^{19}$

Raymond de Vries comments that neuroethicists would do well to understand these different possibilities because if they fail to do so, 'they are less inclined to appreciate the way in which funding sources, and the structure of industry and academic research, shape bioethics and neuroethics'. Against that brief background context, the rest of this chapter is concerned with some examples of what neuroethicists do-particularly in regard to the problem of dual use.

15 Lombera, S. and Illes, J. 2009, 'The international dimension of neuroethics', Developing World Bioethics, vol. 9, no. 2, pp. 57-64.

16 Illes, J. et al. 2005, 'International perspectives on engaging the public in neuroethics', National Review of Neuroscience, vol. 6, no. 12, pp. 977-82; Illes, J. et al. 2010, 'Neurotalk: improving the communication of neuroscience', National Review of Neuroscience, vol. 11, no. 1, pp. 1-20.

17 Wolpe, P. R. 2006, 'Reasons scientists avoid thinking about ethics', Cell, vol. 125, no. 6, pp. 1023-5.

18 Sahakian, B. J. and Morein-Zemir, S. 2009, 'Neuroscientists need neuroethics teaching', Science, vol. 325 , p. 147.

19 Blank, op. cit. 


\section{Case studies}

Whilst the intention here is to concentrate on the ethical issues involved in the practical consequences of the advances in neuroscience, it should be made clear that this is not because we underestimate the importance of the philosophical issues raised by modern neuroscience. This point has been made regularly in reviews of the emerging field: an inadequate model of the brain and mind is likely to cause great misunderstandings and practical difficulties. ${ }^{20}$ For example, if someone is in a persistent vegetative state but neuroimaging shows that he or she is able to respond to some questions, ${ }^{21}$ can informed consent be achieved for treatment? Clearly, the brain is not without response, but is there an ability to decide on complex questions?

If such questions at the more philosophical end of the spectrum of the empirical issues are set aside, it is clear that neuroethicists have considered questions arising from three types of new technologies: neuroimaging; pharmacological enhancement; and non-pharmacological enhancement (such as brain-machine interfaces, transcranial magnetic stimulation, and direct current stimulation). There is also now some consideration being given to how the combination of these technologies with genomics ${ }^{22}$ and information technology ${ }^{23}$ may affect the ethical questions.

Some of the ethical questions that arise with these new technologies are far from new. In this regard neuroethics has carefully noted the issues relating to safety of the technologies, the validity of results drawn from complex analyses derived from neuro-'imaging' techniques, and how these images are understood by the media and general public. Neuroethicists have also discussed the obligations that researchers have for their findings - for example, what to do about findings that suggest that the person involved could have an increased likelihood of illness in the future.

Despite acknowledging these issues that have long-running parallels in bioethics, neuroethicists' writing makes frequent reference to the fact that some of the issues that arise from the application of these new technologies are novel. As

\footnotetext{
20 Glannon, W. 2009, 'Our brains are not us', Bioethics, vol. 23, no. 6, pp. 321-9.

21 Owen, A. M. et al. 2006, 'Detecting awareness in the vegetative state', Science, vol. 313 , p. 1402.

22 Tairyan, K. and Illes, J. 2009, 'Imaging genetics and the power of combined technologies: a perspective from neuroethics', Neuroscience, vol. 164, pp. 7-15.

23 Amari, S.-I. 2002, 'Neuroinformatics: the integration of shared databases and tools towards integrative neuroscience', Journal of Integrative Neuroscience, vol. 1, no. 2, pp. 117-28.
} 
Farah and Wolpe express it: 'The brain is the organ of mind and consciousness, the locus of our sense of selfhood. Interventions in the brain therefore have different ethical implications than interventions in other organs. ${ }^{24}$

With regard to neuroimaging, there are numerous discussions of the possibility, and thus implications, of being able to detect when people are intentionally carrying out a deception. There has been wide discussion of the implications of being able to detect, through neuroimaging, people's desire for certain products and the consequences of the growth of 'neuromarketing'. Concerns have also been expressed about the dangers to privacy if such personal characteristics can be elucidated by neuroimaging.

With respect to pharmacological enhancement, there are again issues of safety, validity and communication of findings to a non-expert audience that are not specific to the concerns of neuroscientists and neuroethicists. Again, however, the neuroethicists' writing clearly indicates that they believe the possibilities of enhancing attention, memory and mood and the reverse possibility of damping down memories (to help people suffering from post-traumatic stress disorder) do raise new ethical issues. What happens, for example, to those who choose not to be enhanced, or who cannot afford to be enhanced, in an enhancementridden society? And, again touching on the philosophical, what happens to our sense of self and worth if we can have a better attention span, memory or mood not by work to achieve such developments but by 'popping a pill'?

A similar set of novel and not so novel issues arises in regard to non-pharmaceutical enhancement, but one unusual point can be noted in the concerns expressed about military funding of work on brain-mind interfaces and of 'new breeds of cyborgs'. Nevertheless, it is clear that this worry about the military implications of the advances in neuroscience and related technologies is very limited in the neuroethics literature. The limited nature of the neuroethical debate was noted by Joelle Abi-Rached in her discussion of the launch of the European Neuroscience and Society Network. In her view, this has to change and the debate 'must also include the controversies surrounding the potential application of various technologies in "neurosecurity" and "counter-terrorism"". ${ }^{25}$

She correctly references Jonathan Moreno's book Mind Wars: Brain Research and National Defense ${ }^{26}$ when making her point. Even there, however - in the only extended discussion of the possible misapplications of various technologiesthere is very little discussion of the problem of dual use. Indeed, although in

24 Farah, M. J. and Wolpe, P. R. 2004, 'Monitoring and manipulating the human brain: new neuroscience technologies and their ethical implications', Hastings Center Reports, vol. 34, no. 3, pp. 35-45.

25 Abi-Rached, J. M. 2008, 'The implications of the new brain sciences', EMBO Reports, vol. 9, no. 12, pp. $1158-62$.

26 Moreno, J. D. 2006, Mind Wars: Brain Research and National Defense, Dana Press, Washington, DC. 
a 2007 editorial in Science, Henry Greely's reflections on neuroethics included the view that '[o]ther studies may have military implications: Suppose brain stimulation created an indefinitely awake and alert soldier or pilot? Will neuroscience be a new source of dual-use technologies such as those we worry about for biological or chemical warfare?' (emphasis added). ${ }^{27}$

It is very difficult to find even a reference to, let alone a discussion of, the problem of dual use in the neuroethics literature, even when it deals with national security issues. ${ }^{28}$

For those trained in natural science, a further striking feature of the neuroethics literature is the generality of the discussion around the ethical implications of neuroscience. ${ }^{29}$ There is very good reason to believe that the ongoing advances in civil neuroscience could produce materials, technologies and knowledge that could later be applied, for example, to develop new forms of incapacitating chemicals for use in the 'War on Terror'. As well, they could lead to the erosion and eventual demise of the prohibition on the use of the modern life sciences for non-peaceful purposes that is embodied in the Biological and Toxin Weapons Convention and the Chemical Weapons Convention. ${ }^{30}$ So what are civil neuroscientists to do to help protect their work from such misuse? Does it perhaps conform to the paradigm described by Lawrence Schmidt and Scott Marratto:

The technological imperative has opened every aspect of human life to relentless transformation. The assumption is that it is always ethically acceptable to experiment to find out whether we can do something, and if we can, we ought to. But the adoption of the technological imperative has meant the liberation of means from ends. ${ }^{31}$

That, of course, is a huge question, but one way to approach it, perhaps, is to look more closely at one of the technologies that neuroethicists have focused upon: cognitive enhancement.

\footnotetext{
27 Greely, H. 2007, 'Editorial: neuroethics', Science, vol. 318, p. 533.

28 Conli, T. et al. 2007, 'Neuroethics and national security', The American Journal of Bioethics, vol. 7, no. 5, pp. 3-13; Resnik, D. B. 2007, 'Neuroethics, national security and secrecy', The American Journal of Bioethics, vol. 7 , no. 5 , pp. 14-26.

29 See, for example, Illes, J. 2007, 'Empirical neuroethics', EMBO Reports, vol. 8, pp. S57-60, Figures 2 and 3; and Glannon, op. cit., Figure 2.

30 See <www.opbw.org $>$ and <www.opcw.org $>$.

31 Schmidt, L. E. and Maratlo, S. 2008, The End of Ethics in a Technological Society, McGill-Queen's University Press, Montreal and Kingston.
} 


\section{Cognitive enhancement}

In December 2008, Nature carried a commentary by Henry Greely and five colleagues titled 'Towards responsible use of cognitive-enhancing drugs by the healthy'. The stated aim of this piece was to 'propose actions that will help society accept the benefits of enhancement, given appropriate research and evolved regulation' ${ }^{32}$

Arguing that these drugs are just another means devised by our innovative species to improve itself, they suggested cognitive-enhancing drugs should 'be viewed in the same general category as education, good health habits and information technology'. They listed what they viewed as standard arguments against the use of these drugs - cheating, unnaturalness and drug abuse - and dismissed them. They did accept, however, that questions of safety, freedom (from coercion to enhance) and fairness (towards those who could not afford enhancement) would need to be addressed. They also suggested a program of research, professional guidance, public understanding and regulation development be undertaken so that responsible use was facilitated. In a direct critique of this commentary, two Canadian neuroethicists struck a much more cautious note, arguing that the question of safety was far from settled: 'it is important to stress that sizeable gaps exist in our current understanding of the effects, both positive and negative, of neuropharmaceuticals on healthy individuals. ${ }^{33}$

And they pointed out that the simple tasks studied so far in laboratories 'do not reflect the complexity and diversity of activities in learning and thinking'. They thus implied that there was a degree of overstatement amongst those favouring cognitive enhancement in the same way that gene therapy had been oversold by supporters. A more neutral term than cognitive enhancement, they suggested, might be 'non-medical use of prescription drugs'. These critics also had concerns about the impact on health resources of interest in cognitive enhancement - for example, about the safety research program suggested by advocates eating up resources that were more urgently needed elsewhere, for example, in treating people who are ill. In short, there does not yet appear to be a settled view amongst neuroethicists about cognitive enhancement. ${ }^{34}$

Yet there is a considerable literature on this subject and certainly enough to ask two questions relevant to dual use: 1) do neuroethicists dealing with cognitive

32 Greely, H. et al. 2008, 'Towards responsible use of cognitive-enhancing drugs by the healthy', Nature, vol. 456, pp. 702-5.

33 Racine, E. and Forlini, C. 2009, 'Expectations regarding cognitive enhancement create substantial challenges', Journal of Medical Ethics, vol. 35, pp. 469-70.

34 Harris, J. and Chatterjee, A. 2009, 'Head to head: is it acceptable for people to take methylphenidate to enhance performance?’ BMJ, vol. 338, pp. 1532-3. 
enhancement consider the issue of dual use, and 2) do the investigations of the mechanism of enhancement by neuroscientists indicate any awareness of the possibility that their work could be used for the very opposite manner by those with malign intent?

Most discussions of cognitive enhancement are concerned with the use of drugs such as: methylphenidate (used medically to help people suffering from attention deficit hyperactivity disorder) to improve attention; modafinil (used medically to help people suffering from sleep problems to improve alertness; and SSRIsselective serotonin reuptake inhibitors (used medically to help people suffering from problems of mood) - to improve the mood of people who are unwell. A particularly interesting subject in regard to this chapter is the use of the drug propranolol, not to improve memory but to help people to forget emotionally laden traumatic events - memories that recur in post-traumatic stress disorder (PTSD). ${ }^{35}$

In such discussions it is certainly possible to find what appears to be approval of military-funded research that could fundamentally change war-fighting and force employment because of, for example, the elimination of the need for sleep and the maintenance of a high level of cognitive performance. ${ }^{36}$ On the other hand, it is rare indeed to find a clear recognition in the neuroethics literature of the problem of dual use in regard to cognitive enhancement. As Kathinka Evers pointed out:

It has been suggested that therapeutic forgetting is interesting for military purposes, for example, to provide soldiers with propranolol before a battle. A problem here is that if it helps them forget what they have been subjected to it also helps them forget what they have done to others. ${ }^{37}$

This comment is clearly a special case because Evers is one of the few bioethicists who has addressed the general issue of dual-use bioethics. ${ }^{38}$

If we turn to the neuroscience, it is clear that safety issues involved in cognitive enhancement are sometimes well understood. As Cakic recently pointed out: ‘For ... psychostimulants such as methylphenidate, the dangers are real and

\footnotetext{
35 Glannon, W. 2008, 'Psychopharmaceutical enhancement', Neuroethics, vol. 1, pp. 45-54.

36 Sahakian, B. J. and Morein-Zamir, S. 2010, 'Neuroethical issues in cognitive enhancement', Journal of Psychopharmacology [epub ahead of print, doi: 10.1177].

37 Evers, K. 2007, 'Perspectives on memory manipulation: using beta-blockers to cure post-traumatic stress disorder', Cambridge Quarterly of Healthcare Ethics, vol. 16, pp. 138-46.

38 Research Ethics and Bioethics, University of Uppsala, <www.crb.uu.se>.
} 
relatively well known. Aside from its abuse potential, methylphenidate may aggravate mental illness, produce sleep disturbances and is associated with cerebrovascular complications. ${ }^{39}$

It is also clear that we know a great deal about how emotion-laden memories are laid down in mammals and the way in which propranolol can be used to interfere with the role of noradrenaline in the consolidation and reconsolidation of such memories. ${ }^{40}$

Whilst accepting that some modest improvements in capabilities may be achieved, recent detailed reviews of the science and ethics of cognitive enhancement have emphasised caveats: 'first ... doses most effective in facilitating one behavior could at the same time exert null or even detrimental effects on other cognitive domains. Second, individuals with "low memory span" might benefit from cognitive-enhancing drugs, whereas "high span subjects" are "overdosed". ${ }^{41}$

And, finally: 'evidence suggests that a number of trade-offs could occur. For example, increases of cognitive stability might come at the cost of a decreased capacity to flexibly alter behaviour.'

This particular review also discusses six ethical issues found in the literature: safety; societal pressure; fairness and equality; enhancement versus therapy; authenticity and personal identity; and happiness and human flourishing. In the last of these it does refer to the possibility that the blunting of memory could involve violation of 'a duty to remember and bear witness of crimes and atrocities'. Thus it touches on the question of dual use, but again there is no indication that the general point - that all of the work in cognitive enhancement could be dual use- - has been understood.

Another recent review that notes the problem of such misuse in the blunting of memories without drawing the general conclusion about dual use does make the crucial point that our knowledge of the brain remains limited: 'the fundamental question is: are we technically ready and do we have sufficient basic knowledge to develop such drugs without risking a deadly brain doping? ${ }^{42}$

A possible response is to say that cognitive enhancement has been much overhyped and that major brain modifications are years away and therefore

39 Cakic, V. 2009, 'Smart drugs for cognitive enhancement: ethical and pragmatic considerations in the era of cosmetic neurology', Journal of Medical Ethics, vol. 35, pp. 611-15.

40 Tully, K. and Bolshakov, V. Y. 2010, 'Emotional enhancement of memory: how norepinephrine enables synaptic plasticity', Molecular Brain, vol. 3, pp. 15-24; Dando, M. R. 2007, 'Scientific outlook for the development of incapacitants', in Pearson et al., op. cit., pp. 123-48.

41 de Jongh, R. et al. 2008, 'Botox for the brain: enhancement of cognition, mood and pro-social behavior and blunting of unwanted memories', Neuroscience and Biobehavioral Reviews, vol. 32, pp. 760-76.

42 Lanni, C. et al. 2008, 'Cognitive enhancers between treating and doping the mind', Pharmacological Research, vol. 57, pp. 196-213. 
we should not worry too much about the state of neuroethics and its lack of coverage of the problem of dual use. That is indeed what one neuroscientist stated recently in a review of a book of essays on neuroethics: 'the participants in this discussion often claim that their speculative approach provides us with the unique opportunity to discuss the ethical consequences of new technologies before they are fully developed ... However, do we really need a debate on a technology that will probably never materialise? ${ }^{\prime 43}$

In this view, we do not really need to be concerned about neuroethics because the debate amongst neuroethicists is not of great importance. Many people took amphetamines during and after World War II so the present uses of drugs for cognitive enhancement is nothing new and, given the limited benefits that are likely to be available for the foreseeable future, the present phase will surely pass quietly away.

\section{Back to dual use}

The problem with the benign viewpoint voiced above (which does not take into account malign misappropriation of neuroscience advancements) is that whilst it may be appropriate for such civil uses of cognitive enhancement, it surely cannot be said to apply to the problem of military dual use. Here, in addition to a long history of misuse of the ongoing advances in neuroscience, we clearly have evidence of state-level development and use of novel chemical incapacitants. Furthermore, there is an obvious concern that continued interest in such developments could lead to the erosion and, potentially, the destruction of the prohibition of chemical and biological weapons embodied in the Chemical Weapons Convention and the Biological and Toxin Weapons Convention.

It is not as if it is difficult to find evidence of real concern amongst experts about this issue. For example, when the International Committee of the Red Cross launched its appeal on 'Biotechnology, Weapons and Humanity', the eminent neuroscientist Professor Tamas Bartfai worried about the dangers of the misuse of advances in neuroscience. He noted that the acetylcholine system had been the target of successive generations of lethal nerve gases and for drugs designed to help people suffering from Alzheimer's disease, and went on to point out a number of clear-cut ways in which current developments in the neurosciences might be subject to misuse. ${ }^{44}$ Similarly, Robert McCreight, a senior US civil servant, gave a lecture on the implications of the advances in neuroscience for

43 Quednow, B. B. 2010, 'Ethics of neuroenhancement: a phantom debate', [Books Forum], BioSocieties, vol. 5, no. 1, pp. 153-6.

44 Bartfai, T. and Sellstem, A., 'Neurobiology - weapons and humanity', Presentation at the launch of the appeal on Biotechnology, Weapons and Humanity, International Committee of the Red Cross, Montreux. 
national security and future strategic weapons in January 2007. As he noted: 'Scientific research, concept development, examination of bioethical issues related to enhanced mental health and considerable long term funding support has been ongoing for several years in the broad area of neuroscience. ${ }^{\prime 45}$

Then he asked: 'Have we adequately analysed and discussed the dual-use implications of neuroscience, particularly its various military applications, and the extent to which operational safeguards and societal controls are needed to manage or control its most destructive weapons outcomes or debilitating systems?'

McCreight did not think that there had been adequate analysis and discussion and suggested that unless measures were taken 'we may face new categories of weapons before 2010 held by several nations both friendly and hostile'.

Certainly, some practising neuroscientists have raised concerns about the hostile misuse of advances in neuroscience, ${ }^{46}$ and there are a few bioethicists who have tried to contribute to a better understanding of the problem of dual use. ${ }^{47}$ Given this relatively early stage in the development of neuroethics, it is not clear whether neuroethicists will have anything special to add to the work of bioethicists in general, but if they do aspire to help as 'architects of moral space' by 'fostering open and constructive dialogue, discussion and debate' about critical issues, ${ }^{48}$ in order to develop 'smarter regulation' where that is necessary ${ }^{49}$ then surely there is no better time than now to begin to develop their view of the neuroethics of dual use.

\section{Conclusion}

The field of neuroscience has grown considerably in the past decade. Advances in neuroscience already have raised important questions on a wide range of policy issues, such as those affecting neurotoxins and the environment, mental health, child development, cognitive enhancement, criminal behaviour, the safety and efficacy of pharmaceuticals and medical devices, and the ethics and regulation of emerging discoveries. To an ever-increasing understanding of the

\footnotetext{
45 McCreight, R. 2007, ‘Protecting our national neuroscience infrastructure: implications for homeland security', Presentation to National Security and the Future of Strategic Weapons, George Washington University, Institute of Crisis, Disaster and Risk Management, $<$ http://www.chds.us/?fs:file \&mode=dl\&drm= $. . \% 2 \mathrm{~F} . . \% 2$ Fresources $\% 2$ Fsummit $\% 2 \mathrm{~F} \% 2$ Fsummit07\&f=McCreight-GeorgeWashUniv.ppt\&altf=McCreightGeorgeWashUniv.ppt>.

46 Bell, C. 2010, 'Neurons for peace: take the pledge, brain scientists', New Scientist, vol. 2746 (8 February).

47 Atlas, R. M. 2009, 'Responsible conduct by life scientists in an age of terrorism', Science and Engineering Ethics, vol. 15, pp. 293-301.

48 Robert, J. S. 2009, 'Toward a better bioethics', Science and Engineering Ethics, vol. 15, pp. 283-91.

49 Sutton, V. 2009, 'Smarter regulations', Science and Engineering Ethics, vol. 15, pp. 303-9.
} 
brain mechanisms associated with core human attributes and values should also correspond an increasing interest in the possible dual-use implications of such advancements, giving the various ways, not always benign, in which the new knowledge could be used. Neuroethics addresses the various philosophical issues around the relationship between brain and mind as well as practical issues about the impact upon society of our ability to understand and manipulate the brain. The problem of dual use, consisting in a malign appropriation of knowledge initially designed for benign purposes, should be an important focus of neuroethicists' analyses. In particular, the long-term applications and impacts of neuroscience are likely to be powerful and profound. As pointed out by Marchant and Gulley: 'Military and intelligence agencies, with the most at stake from such applications in terms of both benefits and risks, recognize the potential of neuroscience to revolutionize intelligence gathering and warfare. ${ }^{50}$

Furthermore, as pointed out by Jonathan Moreno, the 11 September 2001 attacks have resulted in increased efforts to exploit all technical possibilities for enhancing security. The Pentagon's Defense Advanced Research Projects Agency (DARPA) is supporting work at Lockheed Martin on remote brain prints and the scientist in charge already claims to be able to tell if a person is thinking of a certain number. In the words of Moreno, 'a striking aspect of much of this and other national security work being done in the field of neuroscience is that it is "dual use" - potentially applicable to medical therapy or other peaceful purposes as well as combat, riot control, hostage situations, or other security problems'. ${ }^{51}$

Worryingly, from a preliminary review of the literature on neuroethics, it clearly emerges that, while publications abound on issues such as lie detection, informed consent for certain patients and around the implications of neuroimaging, the problem of dual use is very marginally addressed.

In the majority of research centres and institutions, no mention is made of the problem of dual use, and the overwhelming assumption is that neuroscience works for the betterment of humanity worldwide (see Appendix A). If this is what neuroethicists are working for, more attention should surely be devoted to the way advances in neuroscience could be misused. Leading neuroethicists also very rarely address the issue of dual use in neuroscience, with few notable exceptions. $^{52}$

50 Marchant, G. and Gulley, L. 2010, 'National security neuroscience and the reverse dual-use dilemma', АЈОВ Neuroscience, vol. 1, no. 2, pp. 20-2.

51 Ibid.

52 Moreno, J. 2005, 'Dual use and the "moral taint" problem', The American Journal of Bioethics, vol. 5, no. 2, pp. 52-3; Moreno, J. 2008, 'Using neuro-pharmacology to improve interrogation techniques', Bulletin of the Atomic Scientists; Huang, J. Y. and Kosal, M. E. 2008, 'The security impact of the neuroscience', Bulletin of the Atomic Scientists. 
Nevertheless, if neuroethicists have paid almost no attention to the problem of dual use, the issue should be of great concern to both neuroscientists and neuroethicists, who should critically approach dual use in neuroscience in order to save neuroscience from dreadful distortions of its intended purpose. In the current environment, a similar remarkable omission, if not addressed urgently, will be soon deplored. 